\title{
Physics Validation of LHC Simulations
}

\author{
Alberto Ribon* \\ CERN, Switzerland \\ E-mail: Alberto.Ribon@cern.ch
}

\begin{abstract}
A review of the strategy and the results for the physics validation of Geant4 and Fluka detector simulation engines for LHC applications is presented. The main focus is on hadronic physics, where most of the efforts are currently concentrated. In particular, comparisons between simulation and test-beam data for the most relevant calorimeter observables, for different experiments and detector technologies, are discussed.
\end{abstract}

International Europhysics Conference on High Energy Physics

July 21st - 27th 2005

Lisboa, Portugal

${ }^{*}$ Speaker. 


\section{Introduction}

Hadronic physics is notoriously a very broad and difficult field, mainly because the underlying theory, Quantum Chromodynamics (QCD), cannot produce predictions for observables whose dominant energy range is outside the perturbative high-energy regime. The only current viable approach, in these cases, is to use different simplified models, whose approximated validity is often restricted to particular incident particles, target material types, and interaction energies. By using a proper set of these models it is often possible to cover different regions of interest.

In Geant4 [1] a large set of hadronic models are available, and users can choose and combine them according to their needs, in terms of application, precision, and computing time. To ease such a choice, a certain number of "educated guess" Physics Lists, each being a complete and consistent collection of different models, are provided according to use cases.

The goal of this paper is to report about the validation of some of these Geant4 Physics Lists (LHEP, QGSC, QGSP, QGSP_BIC, QGSP_BERT), and also of FLUKA simulation engine [2], in the context of the LHC experiments. We will focus here only on the hadronic physics validation (the electromagnetic physics has been already validated at the percent level).

There are two complementary approaches to the physics validation of simulation results: one is based on thin-target setups with simple geometries, that allow to test, in a clean and simple environment, single interactions or effects; the other one relies on calorimeter test-beam setups, in which the observables are the convolution of many complex processes and interactions.

We will treat here some of the work that has been carried out in the context of the LCG (LHC Computing Grid Simulation) Physics Validation Project [3], starting first with three thin-target tests, and then with three calorimeter test-beams.

\section{Pixel test-beam}

A test-beam with $180 \mathrm{GeV} / \mathrm{c}$ positive pions on silicon sensors of the ATLAS Pixel tracker detector has been made at CERN in 2001 [4]. It consists of a telescope of four microstrip planes (each double sided), two pixel detector planes (to test two different chips), and a scintillator counter. In a run dedicated to the study of hadronic interactions, the trigger required an energy deposition in the scintillator corresponding to at least three minimum-ionising particles. A total of about 800000 interaction events were collected. Only those events in which at least three clusters have been reconstructed in each of the three downstream microstrip planes (in both sides) are further considered in the analysis. We refer to the note [5] for more details. The conclusion is that both Geant 4 and FLUKA describe reasonably well the test-beam data, more or less at the same level, although there are some discrepancies in some observables.

\section{Neutron production cross-sections}

Neutron production from proton bombardment, and in particular $(p, x n)$ double-differential cross-section measurements, $d^{2} \sigma / d \Omega d E_{n}$, i.e. neutron spectrum at fixed angles, are an important benchmark for the validation of hadronic models. Here we consider measurements made at Los Alamos Meson Physics Facility (LAMPF) for proton beam energies of 113, 256, 597, and $800 \mathrm{MeV}$, 
and for angles of $7.5^{\circ} .30^{\circ}, 60^{\circ}, 120^{\circ}$ and $150^{\circ}$ [6]. For details, plots and further references we invite to see the note [7]. The preliminary conclusions from the comparison of the simulation results with the experimental data are the following. While the Geant4 LHEP Physics List is clearly not suitable for the simulation of $(p, x n)$ double-differential cross-sections, FLUKA and Geant4 QGSP_BERT and QGSP_BIC Physics Lists do all reproduce the double-differential crosssection data measured by Los Alamos at the level of $20 \%$ to $50 \%$.

\section{In-flight pion absorption}

Data [8] of thin-target experiments on in-flight $\pi^{+}$and $\pi^{-}$absorption are compared with the Geant 4 and FLUKA simulations. Pion beam energies are between $23 \mathrm{MeV}$ and $315 \mathrm{MeV}$, and targets are made of $\mathrm{Al}, \mathrm{Cu}$ and $\mathrm{Au}$. The in-flight pion absorption process can affect in particular the $e / h$ ratio of calorimeters, as well as the energy resolution at low and medium energies, and therefore needs to be well understood for the simulation of LHC calorimeters. The results reported in [9] show a generally good agreement between both simulation packages and the experimental data. Unfortunately, due to large uncertainties in the data, precise comparisons are not possible. Geant 4 and FLUKA agree better with each other for intermediate weight materials like $\mathrm{Cu}$. The discrepancies become larger for light materials like Al and for the heavy materials like Au. On the other hand, the multiplicity and type of the outgoing particles are qualitatively similar, indicating that the implementation of the processes is correct.

\section{Calorimeter test-beams}

We discuss here the results of the comparisons between Geant4 simulation, with LHEP and QGSP Physics Lists, with three different calorimeter test-beam data. The first calorimeter testbeam setup is the ATLAS HEC (Hadron End-Cap) [10], which is a sampling calorimeter with copper as absorber and liquid argon as sensitive part. The second calorimeter test-beam setup is the ATLAS Tilecal [11], which is the central (barrel and extended barrel) hadronic calorimeter, a sampling calorimeter made of iron as absorber and scintillator tiles as active medium. The third calorimeter test-beam setup is the combined CMS ECAL+HCAL [12]. The electromagnetic part is a matrix of $\mathrm{PbWO} 4$ crystals, whereas the hadronic part is a sampling calorimeter made of copper as absorber and scintillator tiles as sensitive part. The whole apparatus is embedded in a magnetic field of max 3 Tesla. The results [13] of these comparisons show that the energy resolution and the $e / \pi$ is well reproduced by the Geant4 Physics Lists LHEP and QGSP, whereas showers are shorter and narrower than the real ones, especially for QGSP.

\section{Conclusions}

We have presented several validation tests for Geant4 Hadronic Physics, some of which also showing the comparison with FLUKA simulation. This work concludes the first round of hadronic physics validation, with good results. The conclusion is that Geant4 LHEP and QGSP Physics Lists are in good agreement with experimental data, for most observables, and in particular for the calorimeter energy resolution and for the $e / \pi$ ratio, essentially matching the physics requirements 
[14]. These are coming from the demand that the dominant systematic uncertainties for all physics analyses should not be due to the imperfect simulation. Longitudinal and transverse shower shapes, however, are not reproduced very well by Geant4, in particular for QGSP Physics List. Work is in progress to improve them, and more comparisons are expected from the test-beams made in 2004. Another area of interest, in progress, is the one of Geant4 background radiation studies in the LHC caverns.

\section{Acknowledgments}

I am pleased to acknowledge the help, assistence, and collaboration of the Geant 4 team (J. Apostolakis, G. Folger, M. Kossov, J.P. Wellisch), the FLUKA team (A. Ferrari, P. Sala), and of all the people who have contributed to the LCG Physics Validation Project (F. Gianotti, W. Pokorski. M. Gallas, J. Beringer, C. Alexa, S. Banerjee, T. Carli, S. Constantinescu, G. Daquino, S. Dita, A. Dotti, D. Elvira, A. Kiryunin, A. Lupi, C. Roda, D. Salihagic, P. Schacht, P. Strizenec, D. Barberis, M. Cervetto, B. Osculati).

\section{References}

[1] S. Agostinelli et al, Geant4 Collaboration, Nuclear Instruments and Methods in Physics Research, A 506 (2003) 250. See also the Geant4 web page: http://cern.ch/geant4 .

[2] A. Fassò, A. Ferrari, J. Ranft, P. Sala, in proceedings of the MonteCarlo 2000 Conference, Lisbon 2000, Springer-Verlag (2001), 159-164, 955-960. See also the FLUKA web page: http://www.fluka.org .

[3] Physics Validation Project, http://lcgapp.cern.ch/project/simu/validation/ .

[4] T.Lari et al, "Test beam results of ATLAS Pixel Sensors", in proceedings of the International Workshop on Semiconductor Pixel Detectors for Particles and X-Rays, Carmel 2002.

[5] A. Ribon, Validation of Geant4 and FLUKA hadronic physics with pixel test-beam data, CERN-LCGAPP-2004-09.

[6] M.M. Meier et al., Nuclear Science and Engineering 102 (1989) 310; 110 (1992) 289.

W.B. Amian et al., Nuclear Science and Engineering 112 (1992) 78; 115 (1993) 1.

[7] J. Beringer, $(p, x n)$ Production Cross Sections: A Benchmark Study for the Validation of Hadronic Physics Simulation at LHC, CERN-LCGAPP-2003-18.

[8] D. Ashery et al, Physcal Review C, 23 (1981) 2173.

K. Nakai et al, Physical Review Letters, 44 (1980) 1446.

[9] W. Pokorski, In-flight Pion Absorption: Second Benchmark Study for the Validation of Hadronic Physics Simulation at the LHC, CERN-LCGAPP-2004-11.

[10] ATLAS Collaboration, Liquid Argon Calorimeter Technical Design Report, CERN/LHCC/96-41 (1996).

[11] ATLAS Collaboration, Tile Calorimeter Technical Design Report, CERN/LHCC 96-42 (1996).

[12] CMS Collaboration, Hadron Calorimeter Technical Design Report, CERN/LHCC/97-31 (1997).

[13] F. Gianotti et al, Geant4 hadronic physics validation with LHC test-beam data: first conclusions, CERN-LCGAPP-2004-10.

[14] A. De Roeck, F. Gianotti, A. Morsch, W. Pokorski, Simulation physics requirements from the LHC experiments, CERN-LCGAPP-2004-02. 